\title{
El papel del capital humano y el aprendizaje en las microempresas de base social en Baja California
}

\section{The role of human capital and learning in Mexican microenterprises: The experience of the state of Baja California}

Martin Ramírez Urquidy, ${ }^{*}$ Michelle Texis Flores ${ }^{* *}$ y José Gabriel Aguilar Barceló ${ }^{* *}$

\section{Resumen}

Este trabajo tiene como objetivos conocer la estructura del capital humano y el aprendizaje en las microempresas de base social del estado de Baja California y determinar el papel de éste sobre el desempeño microempresarial. Ello con el fin de establecer un diagnóstico sobre su contribución y obtener algunas implicaciones de política pública para el sector en esta temática. Los resultados afirman la importancia del capital humano sobre el desempeño de este tipo de negocios, pues existe una relación entre capital humano y el desempeño medido desde diversas perspectivas. Los resultados también dan cuenta de la heterogeneidad en la distribución del capital humano microempresarial entre localidades, sectores, tipos de empresas y género.

Palabras clave: microempresa, capital humano, aprendizaje, emprendedurismo, empleo.

\begin{abstract}
This work aims at understanding the structure of human capital and learning in low value added microenterprises of Baja California and to determine the role of such factors in microenterprise performance, in order to establish a diagnosis on their contribution and get some public policy implications for the sector. The results confirm the importance of human capital on the performance of these businesses, since there is a relationship between human capital and performance as measured in different ways. The results also account for the heterogeneity in the distribution of microenterprise human capital among locations, sectors, types of business and sex.

Keywords: microenterprise, human capital, learning, entrepeneurship, employment.
\end{abstract}

Recibido el 29 de julio de 2013.

Aprobado el 11 de octubre de 2013.

* Director de la Facultad de Economía y Relaciones Internacionales, Universidad Autónoma de Baja California. Correo electrónico: martinramirez@uabc.edu.mx

** Profesores-investigadores de la Facultad de Economía y Relaciones Internacionales, Universidad Autónoma de Baja California. Correos electrónicos: mtexis@ uabc.edu.mx; gaba@uabc.edu.mx 


\section{Introducción}

La estructura empresarial de Baja California estaba conformada por 80654 unidades económicas en 2008, según el último censo elaborado por el Instituto Nacional de Estadística y Geografía (INEGI). Al igual que las tendencias del resto del país, $99.5 \%$ de dichos establecimientos son micro, pequeñas y medianas empresas, con una presencia fuertemente sesgada hacia las primeras. Las microempresas en México son aquellas unidades productivas que cuentan con entre 1 y 10 empleados incluyendo el dueño. Si bien gran parte de ellas se concentra en el estrato de entre 1 y 5 empleados según la clasificación por número de empleados publicada en el Diario Oficial de la Federación (DOF) en 2002. Este estrato por sí mismo contabiliza $91.5 \%$ de los establecimientos, dato que se ha mantenido desde hace ya varios lustros debido a una importante actividad de creación de empresas, ya que pasan de 54412 unidades en 1999 a 73870 en 2008, un crecimiento promedio anual de $6 \%$. Los datos disponibles en materia de empleo por estrato empresarial derivados de la Encuesta Nacional de Ocupación y Empleo (ENOE) del INEGI, brindan una visión consistente con los datos anteriores. La aportación de las microempresas como porcentaje de la ocupación estatal ha ido en aumento de $41 \%$ en el periodo 2005-2007 a $45 \%$ en 2011. Ello viene a la par con una participación de la pequeña empresa que permanece prácticamente constante entre $22 \%$ y $23 \%$, y una participación de las medianas y grandes empresas que viene a menos. Otro dato importante es que el estrato que aporta más a la creación de los nuevos empleos son igualmente los micronegocios, explicando más del $61 \%$ ya que de los 202 mil empleos generados en el periodo 2005-2011, sólo las microempresas aportaron más de 124 mil, muy por encima de las grandes que no llegan a 17 mil, las medianas con 6.6 mil e incluso las pequeñas que aportaron casi 68 mil empleos.

Dentro del propio estrato microempresarial, se encuentran los micronegocios de base social. Si bien la información de este estrato es limitada dada su naturaleza informal e intermitente, sus formas de organización y las actividades que realizan, el Banco Interamericano de Desarrollo (BID, 1997) las considera como una unidad de producción de subsistencia con bajos niveles de capital y ventas, que normalmente se encuentran al margen del marco regulatorio, cuyo propietario es quien lleva a cabo la mayoría de las 
actividades del negocio, sus empleados suelen provenir del seno familiar y no hacen una separación entre los recursos de la familia y los de la empresa. Estos negocios son llamados también como "microempresas de base social", que constituyen el estrato empresarial más necesitado y pobre del sector económico. El propio BID (2005) sugiere que este tipo de negocios "es probablemente, la muestra más patente de creatividad, dinamismo y adaptabilidad del segmento de la población de menores ingresos de América Latina y el Caribe (...) La microempresa no es sinónimo de pobreza sino, por el contrario, un vehículo importante para que familias de ingresos limitados puedan escapar de la pobreza por medio de actividades productivas regidas por el mercado".

La proliferación de los micronegocios y su aporte al empleo trae la necesidad de promover un mayor entendimiento sobre el estrato a fin de promover políticas públicas en apoyo a este sector. Las estrategias de desarrollo para este estrato han quedado justificadas entre los lineamientos de política recomendados por organizaciones internacionales de desarrollo. De hecho, la promoción de estas empresas ha sido un tema relevante en los informes de desarrollo y los programas de los organismos internacionales como el Banco Mundial, el Banco Interamericano de Desarrollo, la Comisión Económica para América Latina y el Caribe (CEPAL) y la Comisión Europea, que consideran el apoyo a estas empresas como una estrategia de mercado para el alivio de la pobreza.

Este trabajo constituye un esfuerzo por conocer la estructura del capital humano y el aprendizaje en las microempresas del estado de Baja California, y determinar el papel de éste sobre el desempeño microempresarial. Por ello, se analiza el impacto de variables que reflejan el capital humano, tales como la escolaridad formal y la experiencia, sobre distintas variables de desempeño en microempresas consideradas de base social. Se contrasta la hipótesis de que los diferenciales en la acumulación de capital humano en estas empresas se asocian con diferenciales en términos de su desempeño. Un aspecto que resulta novedoso para la literatura económica en México, además de la utilización de información con tal nivel de desagregación, es el tratamiento conjunto de los aprendizajes formales adquiridos en instituciones educativas y los aprendizajes informales adquiridos por la experiencia, ya que los estudios de este tipo suelen considerar únicamente los primeros. La contrastación de esta hipótesis debe 
permitir establecer un diagnóstico sobre la contribución de este factor y obtener algunas implicaciones de política pública para el sector en esta temática.

Los resultados de investigación validan la hipótesis y la importancia del capital humano sobre el desempeño de este tipo de negocios, y son consistentes con lo que se encuentra en la literatura para otro tipo de negocios y/o sectores. Los hallazgos también dan cuenta de la heterogeneidad en la distribución del capital humano microempresarial entre localidades, sectores, tipos de empresas y género en Baja California. Ambos grupos de resultados deben servir para el diseño de políticas públicas y el emprendimiento de acciones.

\section{Marco de referencia sobre el capital humano y el aprendizaje}

\section{Definiciones y categorías del capital humano y el aprendizaje}

Shultz (1960 y 1972) fue el primero en reconocer en la literatura económica que algunos atributos inherentes a las personas, tales como la educación y la salud, podrían ser considerados como capital pues traían beneficios económicos tanto para los individuos como para sus organizaciones. Ello debido al impacto de dichos aspectos sobre las capacidades y habilidades de las personas para trabajar y administrar sus asuntos y los de las empresas que los emplean. De esta forma, Shultz propone estos aspectos como una forma de inversión y sus consecuencias como una forma de capital que como tal mejora el rendimiento del esfuerzo humano.

El capital humano se ha definido tradicionalmente desde la perspectiva de la educación, aunque el concepto es más amplio. Shultz (1961) y Becker (1962) proponen diversas categorías, como la infraestructura y los servicios de salud, que afectan la esperanza de vida, la fuerza, la resistencia, el vigor y la vitalidad de las personas, impactando sus capacidades físicas y mentales para desarrollar trabajo productivo. Otro aspecto es la formación o capacitación en el trabajo, que incluye el aprendizaje organizado por las empresas a partir de programas de entrenamiento; o bien el trabajo artesanal que impacta necesidades específicas de las empresas y su productividad. Otras categorías son la educación formal organizada 
en las instituciones educativas, los programas de estudio para los adultos y los programas de extensión organizados por instituciones educativas en apoyo a la sociedad.

La inversión en capital humano es concebida por los teóricos del capital humano como los recursos invertidos en las personas, pero también se ha hecho hincapié en aspectos tales como la capacitación laboral, el nivel de escolaridad y otros conocimientos, cuyos rendimientos pueden ser capturados por los empleados en salarios más altos y por las empresas en mayor productividad (Becker, 1975). Este tipo de inversión incrementa la productividad futura de empleo, estimulando el aprendizaje de nuevas habilidades y el mejoramiento de las antiguas. Ello, aparte de las externalidades positivas generadas a escala social.

En cuanto a la educación, según Becker (1975), es un proceso de enseñanza formal en las escuelas, mismas que se especializan en la producción de entrenamiento, diferenciado de las empresas, que ofrecen formación vinculada con la producción de bienes. Según Becker, la formación en el empleo y la educación escolarizada se complementan pues el dominio de algunas técnicas requiere la especialización y la práctica, así que el aprendizaje tiene que ser adquirido tanto en la escuela como en el trabajo. Sin embargo, la acumulación de capital humano no sólo es producto de la inversión en entrenamiento formal en la escuela o en las empresas, sino también de los procesos informales de aprendizaje por experiencia dentro del mismo trabajo (Mungaray y Ramírez, 2007), lo que es conocido también como learning-by-doing o "aprender haciendo". En este sentido, el aprendizaje se define como el proceso que conduce a la acumulación de destrezas que impacta positivamente el desempeño de los trabajadores.

Por último, otra forma de capital humano incluye las transferencias intergeneracionales de las habilidades, el espíritu empresarial, la experiencia, la reputación, los recursos, así como otras de gestión que suceden en el seno familiar y que se derivan de los emprendimientos de sus miembros, particularmente de los padres, y de la exposición al ambiente empresarial (Parker y Robson, 2004). Estas transferencias pueden llegar a ser incluso más importantes en el desempeño de los emprendimientos que las transferencias de capital financiero, tal como algunos teóricos lo han señalado. En particular, los efectos de este tipo de acumulación de capital humano 
se presentan tanto en la probabilidad de que un individuo se convierta en empresario como en las posibilidades de éxito.

\section{Efectos del capital humano y el aprendizaje sobre el desempeño de las microempresas}

Estudios a nivel empírico que relacionan el capital humano con algunas variables de desempeño que aplican a las pequeñas unidades productivas se han centrado en el análisis de su relación con diversos aspectos del emprendimiento. Un primer conjunto de trabajos corresponde a Fane (1975) y Huffman (1977), quienes sugieren que la capacidad de asignación de recursos - es decir, el grado de eficiencia con la que los agentes perciben y responden a los cambios del medio ambiente en el que se desenvuelven - está estrechamente relacionada con el capital humano, en particular la inversión en educación y en programas de extensión. El mismo resultado fue encontrado por Stefanou y Saxena (1988), quienes estudiaron el impacto de los programas de educación y extensión sobre la toma de decisiones de los trabajadores en las empresas agrícolas, encontrando que el capital humano había mejorado la eficiencia asignativa y, consecuentemente, sus beneficios. Por su parte, Liedholm (2002) se centra en el crecimiento de la empresa y sugiere que la formación profesional y la experiencia empresarial previa tienen un impacto positivo y significativo en esta variable, mientras que la evidencia con respecto de la educación es mixta. Para México, los estudios que relacionan el capital humano y las variables de desempeño a nivel de empresa no son numerosos, en particular los relativos a los autoempleados y las microempresas. Uno de estos estudios es el de Hernández-Trillo, Pagán y Paxton (2005), quienes estiman la contribución de la educación y la experiencia empresarial en la ineficiencia técnica en microempresas, encontrando efectos negativos y estadísticamente significativos; otro es el de Mungaray, Ramírez-Urquidi, Texis, Ledezma y Ramírez (2008), quienes encontraron efectos positivos en las curvas de costos derivados de los programas de las extensiones de las microempresas.

Otro grupo de estudios se ha centrado en analizar el papel desempeñado por el capital humano en varios aspectos de la formación de la 
empresa y la probabilidad de éxito. Si bien este tema en la economía no ha sido recurrente, las conclusiones de algunos autores señalan que el capital humano mejora la capacidad de gestión de los propietarios de negocios, aumentando por consiguiente la longevidad y la probabilidad de éxito de los negocios (Bates, 1990), por un lado, y por otro, la probabilidad de que los emprendimientos se vuelvan de mayores ingresos (Bates, 1987).

Otra perspectiva del capital humano considera que las transferencias intergeneracionales de capacidades empresariales y disposición al emprendimiento también impactan la dinámica empresarial. Altonji y Dunn (1991) reportan una correlación significativa entre los ingresos de las empresas y las ganancias intergeneracionales de familiares. En esta línea, Laferrère y McEntee (1996) encontraron que la probabilidad de emprendimiento de los hijos de autoempleados o emprendedores es significativamente mayor que la de aquellos cuyos padres tuvieron otras ocupaciones y que dichos efectos pudieran ser incluso mayores que los derivados de factores pecuniarios como el capital financiero, como lo sugieren Dunn y Holtz-Eakin (2000), y que dichos efectos se daban a través de la propia experiencia laboral y éxito de los padres.

En relación con la productividad como variable de desempeño, Barron, Black y Lowenstein (1987), y Bishop (1994), utilizando metodologías similares aplicables a las empresas y las medidas de productividad a partir de encuestas, revelaron que el entrenamiento impartido por el empleador aumentaba la "productividad subjetiva", es decir, la productividad percibida por el trabajador. En la misma línea, Bartel (1989), empleando una muestra de empresas con múltiples establecimientos, encontró que la inversión en entrenamiento incrementa la productividad. También se ha encontrado que en algunas empresas manufactureras la inversión en entrenamiento estaba directamente relacionada con la productividad futura (Bartel, 1992), y que el nivel promedio de educación tenía un efecto positivo y significativo sobre la productividad (Black y Lynch, 1996).

En otra línea de trabajo, algunos estudios han desarrollado la hipótesis de aprender haciendo, es decir, que a medida que el trabajador desempeña sus responsabilidades en el proceso productivo y repite una operación muchas veces, consigue una mayor eficiencia en el desempeño de sus labores, 
proceso que es llamado aprendizaje. En este sentido, Teplitz (1991) sugiere que conforme la actividad es repetida, el trabajador adquiere familiaridad con los procesos básicos de la empresa, generando una mejora en su destreza manual, lo que puede contribuir en la simplificación de procesos que impacten positivamente en el menor tiempo requerido para realizar una tarea, incluso en los niveles de calidad. Según Arrow (1962), este proceso abre la posibilidad de elevar endógenamente la productividad por ese aprendizaje emprendido por los trabajadores al repetir las tareas propias de su trabajo y enfrentarse a los mismos problemas en el proceso productivo, independientemente del nivel de formación escolar o del trabajo que se tenga. Dichos aprendizajes en el trabajo, de acuerdo con Jovanovic y Nyarko (1995), no sólo eleva la productividad independientemente del capital físico existente, sino que constituye un punto de partida de innovaciones en las técnicas de producción y en las formas de organizar y comercializar los productos (Young, 1993).

\section{Resultados de la investigación: Perfil del capital humano y aprendizaje en las microempresas del estado de Baja California}

Esta sección presenta la metodología y los resultados de la investigación. En primer término, se delimita y caracteriza el perfil de las microempresas de la muestra; y se realiza un análisis descriptivo sobre las características del capital humano de la muestra desde distintas perspectivas con base en cuadros analíticos. Posteriormente se aplican pruebas de hipótesis para determinar el impacto del capital humano sobre variables de desempeño microempresarial por grupos de acuerdo con el tipo y el nivel de capital humano. Ello con la finalidad de verificar la diferencia estadística que pudiera presentarse entre los grupos dependiendo de su perfil de capital humano.

\section{Delimitación y características de la muestra de microempresas}

La disponibilidad de datos del segmento microempresarial de base social es limitada, debido a su naturaleza informal e intermitente, a su forma 
de organización y a las actividades que realiza. De igual forma, el sector presenta una heterogeneidad alta que vuelve difícil caracterizarlo. No obstante, se han logrado tipificaciones que lo diferencian respecto de otros segmentos de micro y pequeñas empresas, lo que permite orientar su estudio. El BID, por ejemplo, considera a la microempresa como una unidad de producción de subsistencia, que normalmente se encuentra al margen del marco regulatorio, cuyo propietario realiza la mayoría de las funciones, sus empleados suelen provenir del seno familiar y no hacen una separación entre los recursos de la familia y los de la empresa (Berger, 1997). Estos negocios se caracterizan por realizar actividades económicas diversas, a pequeña escala y organizadas de manera informal, con poca tecnología y asociadas a los rasgos idiosincrásicos y culturales de quienes las realizan y de sus regiones. Se dan a partir de la explotación de habilidades aprendidas de sus antepasados o de otros negocios pequeños, que por lo general han amortizado ex-ante las inversiones y enfrentan pocas barreras a la entrada y salida de las mismas (Aguilar, Ramírez y Barrón, 2007). Hay otros autores, como Mungaray, Ramírez y Texis (2005), y Carpintero (1998) y Ruiz (1995), que también sostienen el carácter unipersonal y familiar, informal y de subsistencia de este tipo de negocios.

Bajo la descripción anterior, el trabajo contó con una muestra de 5524 microempresas localizadas en barrios y colonias populares de zonas urbanas y rurales de Baja California. El procedimiento de selección de las unidades muestrales fue de conveniencia ya que no existe un directorio que permita hacer una selección aleatoria. Los microempresarios fueron encuestados en campo, en las calles, en hogares de barrios marginados puerta por puerta, centros comunitarios y colonias populares, entre otros. Esto debido a que las microempresas de base social producen regularmente dentro de sus domicilios, en lo que se conoce como "talleres familiares", con anuncios modestos sobre la empresa (o incluso sin ellos). Otros microempresarios fueron más visibles al producir en talleres independientes de los hogares, cerca de éstos o en áreas más céntricas de las colonias. Si bien se contactó a 5524 microempresarios, no todos aceptaron dar toda la información o sabían las respuestas, por lo que las frecuencias pueden variar dependiendo de la pregunta requerida.

El cuadro 1 contiene información por municipio y tipo de unidad económica. Del total de microempresas visitadas, $66 \%$ pertenece a Tijuana, $16 \%$ 
Cuadro 1. Microempresas por tipo de unidad económica

\begin{tabular}{|l|c|c|c|c|c|c|}
\hline \multicolumn{1}{|c|}{ Municipio } & Ambulante & $\begin{array}{c}\text { Puesto } \\
\text { en la } \\
\text { vía } \\
\text { publica }\end{array}$ & $\begin{array}{c}\text { Taller } \\
\text { familiar }\end{array}$ & $\begin{array}{c}\text { Taller } \\
\text { independiente }\end{array}$ & Total & (\%) \\
\hline Ensenada & 73 & 97 & 153 & 194 & 517 & $9 \%$ \\
\hline Mexicali & 218 & 125 & 278 & 279 & 900 & $16 \%$ \\
\hline Rosarito & 47 & 6 & 11 & 5 & 69 & $1 \%$ \\
\hline Tecate & 82 & 98 & 111 & 112 & 403 & $7 \%$ \\
\hline Tijuana & 992 & 754 & 1186 & 703 & 3635 & $66 \%$ \\
\hline Total & 1412 & 1080 & 1739 & 1293 & 5524 & $100 \%$ \\
\hline \multicolumn{1}{|c|}{$(\%)$} & $26 \%$ & $20 \%$ & $31 \%$ & $23 \%$ & $100 \%$ & \\
\hline
\end{tabular}

Fuente: Elaboración propia con datos proporcionados por el Centro de Investigación, Asistencia y Docencia de la Micro y Pequeña Empresa (CIADMYPe), Facultad de Economía y Relaciones Internacionales, UABC.

a Mexicali, $9 \%$ a Ensenada, $7 \%$ a Tecate y $1 \%$ a Rosarito. De estas empresas, poco más de una cuarta parte desarrolla actividades microempresariales como ambulante, 23\% realiza actividades desde su casa en taller familiar, $20 \%$ tiene puesto en la vía pública, y el resto, poco menos de un cuarto, posee un taller independiente, es decir, fuera del hogar y la vía pública.

En cuanto al sector de actividad, las empresas bajo análisis se concentran mayoritariamente en el comercio (48\%, cuadro 2), desarrollando actividades de compra-venta de abarrotes, alimentos y bebidas, y compraventa de prendas de vestir nuevas y usadas, así como artículos de uso personal, principalmente. De ahí siguen las actividades de servicios, con una participación de $36 \%$ de las empresas totales de la muestra, según el cuadro 2, donde se pueden encontrar actividades como servicios de preparación y venta de alimentos, entre otras. Por último, se encuentran las actividades manufactureras con $11 \%$ del total, donde destaca principalmente la elaboración de productos alimenticios y los textiles, cuero y prendas de vestir. 
Cuadro 2. Microempresas por sector de actividad

\begin{tabular}{|l|c|c|c|c|c|}
\hline Municipio & Comercio & Manufactura & Servicios & $\begin{array}{c}\text { No } \\
\text { especificado }\end{array}$ & Total \\
\hline Ensenada & 244 & 72 & 193 & 8 & 517 \\
\hline Mexicali & 470 & 93 & 319 & 18 & 900 \\
\hline Rosarito & 48 & 3 & 17 & 1 & 69 \\
\hline Tecate & 182 & 33 & 188 & 0 & 403 \\
\hline Tijuana & 1735 & 401 & 1254 & 245 & 3635 \\
\hline Total & 2679 & 602 & 1971 & 272 & 5524 \\
\hline \multicolumn{1}{|c|}{$(\%)$} & $48 \%$ & $11 \%$ & $36 \%$ & $5 \%$ & $100 \%$ \\
\hline
\end{tabular}

Fuente: Elaboración propia con datos proporcionados por el Centro de Investigación, Asistencia y Docencia de la Micro y Pequeña Empresa (ciadmype), Facultad de Economía y Relaciones Internacionales, UABC.

\section{El perfil del capital humano en las microempresas}

\section{Indicadores básicos de capital humano}

En primer lugar, el cuadro 3 presenta tres indicadores básicos de capital humano por municipio, asociados con algunos parámetros de la estadística descriptiva, y que representan dos perspectivas básicas del capital humano: la experiencia y la educación formal expresada en años de escolaridad. Estos indicadores señalan, en términos generales, cuáles son las condiciones entre municipio y variables, tales como la experiencia medida en tiempo de operación de la empresa, la edad y el nivel de escolaridad. En primer lugar, se destaca que la escolaridad representativa de la muestra medida como la mediana, es de secundaria, y la mayor parte de los municipios está en este nivel, con excepción de Mexicali, que llega al nivel de preparatoria. Si se considera la media aritmética es posible establecer una ordenación de municipios respecto de la educación; así, en primer 
Cuadro 3. Indicadores básicos de capital humano por municipio

\begin{tabular}{|c|c|c|c|c|}
\hline Municipio & Estadístico & $\begin{array}{l}\text { Nivel educativo } \\
(*)\end{array}$ & $\begin{array}{c}\text { Experiencia } \\
\text { en el negocio } \\
\text { (Años) }\end{array}$ & $\begin{array}{l}\text { Edad } \\
\text { (Años }\end{array}$ \\
\hline \multirow{4}{*}{ Ensenada } & Media & 2.49 & 1.62 & 36.60 \\
\hline & Desv. Est. & 1.51 & 4.25 & 12.24 \\
\hline & Mediana & 2 & 0 & 37 \\
\hline & Frecuencia & 148 & 508 & 148 \\
\hline \multirow{4}{*}{ Mexicali } & Media & 2.81 & 2.37 & 37.60 \\
\hline & Desv. Est. & 1.51 & 5.42 & 12.60 \\
\hline & Mediana & 3 & 0 & 36 \\
\hline & Frecuencia & 536 & 868 & 536 \\
\hline \multirow{4}{*}{$\begin{array}{l}\text { Playas de } \\
\text { Rosarito }\end{array}$} & Media & 1.88 & 5.95 & 40.35 \\
\hline & Desv. Est. & 0.86 & 6.74 & 8.23 \\
\hline & Mediana & 2 & 4 & 42 \\
\hline & Frecuencia & 17 & 61 & 17 \\
\hline \multirow{4}{*}{ Tecate } & Media & 2.24 & 3.01 & 35.91 \\
\hline & Desv. Est. & 1.31 & 5.22 & 12.68 \\
\hline & Mediana & 2 & 1 & 35 \\
\hline & Frecuencia & 192 & 369 & 192 \\
\hline \multirow{4}{*}{ Tijuana } & Media & 2.09 & 3.58 & 39.03 \\
\hline & Desv. Est. & 1.37 & 5.93 & 12.88 \\
\hline & Mediana & 2 & 1 & 38 \\
\hline & Frecuencia & 1718 & 3447 & 1718 \\
\hline \multirow{4}{*}{ Total } & Media & 2.27 & 3.18 & 38.38 \\
\hline & Desv. Est. & 1.43 & 5.71 & 12.78 \\
\hline & Mediana & 2 & 1 & 37 \\
\hline & Frecuencia & 2611 & 5253 & 2611 \\
\hline
\end{tabular}

(*) $0=$ Sin educación, $1=$ Primaria, $2=$ Secundaria, $3=$ Preparatoria, $4=$ Técnica, $5=$ Licenciatura, $6=$ Posgrado.

Fuente: Elaboración propia con datos proporcionados por el Centro de Investigación, Asistencia y Docencia de la Micro y Pequeña Empresa (CiADMype), Facultad de Economía y Relaciones Internacionales, UABC. 
lugar se encuentra la capital del estado y, consecutivamente, Ensenada, Tecate, Tijuana y Playas de Rosarito. En cuanto a la experiencia en el negocio, medida como los años en que el empresario ha operado su empresa, encontramos que en promedio éstas han operado por 3.18 años, aunque la mediana es de sólo 1 debido a la dispersión en los datos. Playas de Rosarito y Tijuana cuentan con un promedio de 5.95 y 3.58 años como empresario, de ahí le siguen Tecate (3.01 años), Mexicali (2.37) y Ensenada (1.62). En cuanto a la edad, se encuentran microempresarios que en promedio cuentan con 38 años, con una desviación estándar de casi 13 años, por lo que es posible encontrar empresarios de más de 50 años. En este rubro se puede observar que los empresarios mayores se encuentran en Playas de Rosarito y Tijuana, con poco más de 40 y 30 años, respectivamente; les siguen Tecate con 35.91, Ensenada 36.60 y Mexicali 37.60 años de edad.

Es posible observar una relación indirecta entre la edad y la educación; es decir, los municipios con microempresarios mayores, a su vez, son los que exhiben una escolaridad promedio menor, lo que puede explicarse por las propias tendencias educativas del país que han ampliado la cobertura en educación básica entre la población a lo largo del tiempo. También se encuentra la relación positiva entre la edad y la experiencia en el negocio, que interactuando con el dato de educación, que sugiere que a una menor educación le corresponde una mayor edad y experiencia en los negocios, podría estar asociado a un emprendimiento por necesidad debido a menores oportunidades de empleo y una necesidad mayor de encontrar sustento en una microempresa.

Nivel educativo de los dueños de los negocios por municipio

El cuadro 4 muestra el número de microempresas por municipio y nivel educativo de los dueños. En el cuadro se observa que en general $62 \%$ de los microempresarios posee estudios entre los rangos de sin estudios a secundaria, cifra que muestra importantes diferencias entre municipios ya que Playas de Rosarito, Tijuana y Tecate presentan $71 \%, 67 \%$ y $63 \%$, respectivamente, en este rango de estudios; mientras que Mexicali muestra 


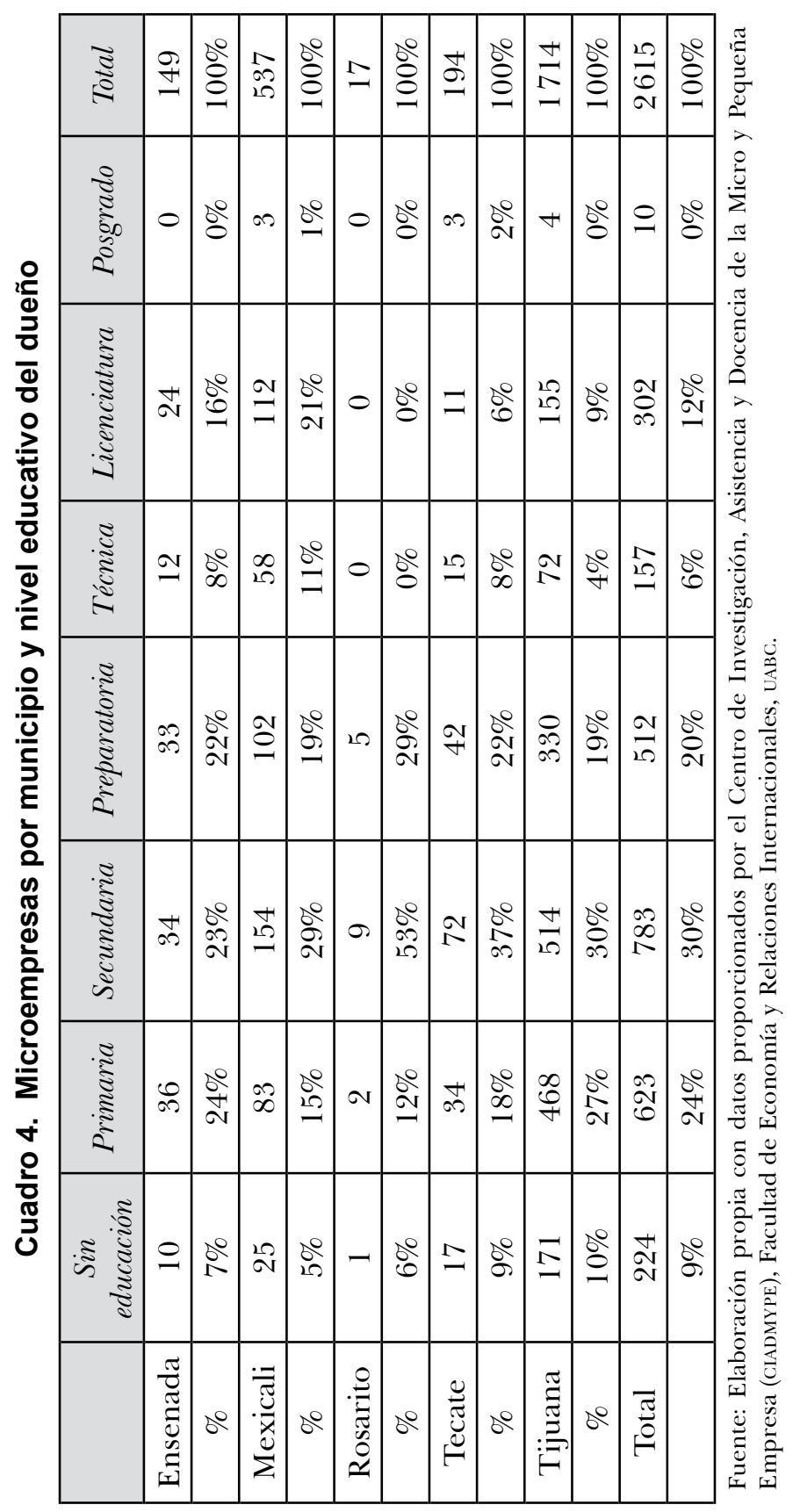


49\% y Ensenada 54\%. Este dato refleja una concentración de los niveles de estudio más bajos en estos tres municipios conurbados.

Los municipios de Ensenada y Mexicali cuentan con una más alta proporción de microempresarios en educación técnica y superior, con una correspondiente menor proporción en los niveles inferiores, Rosarito y Tecate en los niveles de secundaria y preparatoria, y Tijuana en los niveles de primaria y secundaria. El caso de Tijuana merece particular atención no sólo porque registra $10 \%$ de los microempresarios en el nivel de sin estudios y $27 \%$ en primaria, por encima del resto, sino porque además representa $66 \%$ de las empresas de la muestra, alrededor de 1714 . En cuanto al nivel educativo medio-superior, es decir, entre educación preparatoria y técnica, encontramos que todos los municipios, con excepción de Tijuana, cuentan con entre 29\% y $30 \%$ de los microempresarios en este rubro, mientras que Tijuana presenta sólo 23\%, lo que va en el mismo sentido de los datos anteriores. El hecho de que Tijuana y municipios conurbados estén presentando un menor nivel de escolaridad en promedio, pudiera estar asociado con las tendencias de la migración, que en mayor proporción es de individuos con bajos niveles de escolaridad.

Nivel educativo de los dueños de los negocios por sexo

En cuanto al nivel educativo de los microempresarios por sexo, se encuentra una gran similitud entre hombres y mujeres, como ilustra el cuadro 5. En el rubro de sin educación a educación secundaria, encontramos datos que reflejan una ligera desventaja de las mujeres con respecto de los hombres, pues $64 \%$ de ellas se encuentra en este rango, mientras que en el caso de los hombres el promedio es de $61 \%$. En educación media superior ambos grupos poseen $26 \%$ en este rango, mientras que en educación superior se nota la ventaja masculina con 14\%, frente a sólo $11 \%$ de las mujeres.

En general, se observa que la proporción de mujeres en los niveles educativos de sin educación, primaria y secundaria que integran educación básica, es ligeramente mayor, mientras que el de educación superior es mayor para los hombres. En el nivel educativo que integra preparatoria y técnica no existen diferencias entre los grupos. De esta forma, se en- 


\begin{tabular}{|c|c|c|c|c|c|c|}
\hline$\frac{\vec{s}}{\sqrt[0]{0}}$ & 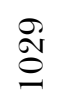 & $\begin{array}{l}\delta^{2} \\
8 \\
0\end{array}$ & $\begin{array}{l}N \\
\infty \\
10 \\
-1\end{array}$ & $\begin{array}{l}0 \\
0 \\
0\end{array}$ & $\begin{array}{l}7 \\
\sigma \\
\sigma\end{array}$ & $\stackrel{8}{0}_{0}^{0}$ \\
\hline 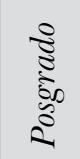 & 0 & $\Delta^{2}$ & $\forall$ & $5_{0}^{0}$ & $\stackrel{\circ}{-}$ & $8_{0}^{\circ}$ \\
\hline 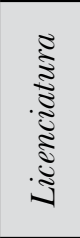 & on & $\begin{array}{l}\text { ô } \\
\text { on }\end{array}$ & $\stackrel{10}{0}$ & 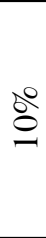 & $\begin{array}{c}\mathcal{O} \\
\text { on }\end{array}$ & $\begin{array}{l}\stackrel{d}{ } \\
\text { on }\end{array}$ \\
\hline 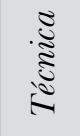 & $\stackrel{\infty}{+}$ & 总 & $\stackrel{g}{\varrho}$ & $\frac{20}{1}$ & in & 80 \\
\hline 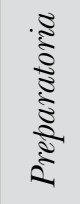 & $\frac{20}{a}$ & $\frac{\partial}{a}$ & $\hat{\mathscr{a}}$ & o̊ & $\frac{O}{20}$ & $\begin{array}{l}0 \\
\stackrel{0}{\circ} \\
\text { o }\end{array}$ \\
\hline 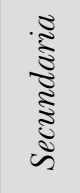 & $\begin{array}{l}\infty \\
\stackrel{\infty}{N}\end{array}$ & $\begin{array}{l}\stackrel{0}{ } \\
\text { oे }\end{array}$ & $\begin{array}{l}\mathscr{1 0} \\
+\end{array}$ & $\frac{b^{2}}{a n}$ & $\begin{array}{l}\infty \\
\infty \\
1\end{array}$ & $\begin{array}{l}0 \\
0 \\
0 \\
0\end{array}$ \\
\hline 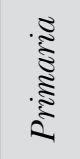 & $\begin{array}{l}\text { के } \\
\text { ๙ి }\end{array}$ & $\begin{array}{l}\text { ô } \\
\text { on } \\
\text { av }\end{array}$ & $\begin{array}{l}+1 \\
\text { on }\end{array}$ & $\frac{\Delta}{d}$ & $\begin{array}{l}\infty \\
\text { v } \\
\tilde{v}\end{array}$ & $\frac{d}{d}$ \\
\hline 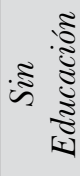 & $\infty$ & $\infty_{\infty}^{0}$ & $\begin{array}{l}\infty \\
\infty\end{array}$ & $\frac{2}{\sigma}$ & $\underset{\sim}{\mathbb{N}}$ & s̊ \\
\hline & 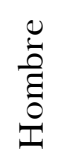 & $\alpha^{e}$ & $\frac{\bar{\omega}}{\underbrace{\Xi}_{\Sigma}}$ & $b^{e}$ & 䒕 & $2^{e}$ \\
\hline
\end{tabular}


cuentra marginalmente una ventaja educativa de los hombres respecto de las mujeres (cuadro 6).

Nivel educativo de los dueños de los negocios por sector de actividad

El análisis del nivel educativo por sector de actividad arroja también algunos datos relevantes. Por un lado, se encuentran las actividades de comercio que cuentan con $9 \%$ de sus empresarios en el rubro de sin educación, cifra similar a la manufactura, mientras que los servicios cuentan con $7 \%$ en este rango educativo. En cuanto a la educación primaria se encuentran diferencias importantes, pues mientras que la manufactura y los servicios tienen $20 \%$ en este rubro educativo, el comercio reportó una cifra de $28 \%$ (cuadro 7). En los niveles de secundaria, preparatoria, técnica, licenciatura y posgrado por separado, no se observan diferencias pronunciadas, sino hasta agruparlos en educación básica, media superior y superior. Se puede observar que la proporción de los microempresarios con educación básica es superior en el comercio, con 58\%, que en los otros dos sectores, que presentan una proporción de $50 \%$. Cuando se trata de educación media-superior, la proporción se invierte, pues para el comercio la proporción sólo llega a $22 \%$ mientras que para la manufactura y los servicios es de 28 y $29 \%$ respectivamente. Los niveles extremos desfavorecen al sector comercio, por un lado, pues como se ilustró anteriormente, cuentan con la mayor proporción de microempresarios sin educación y educación básica y la menor en educación media superior, aunque en la educación superior mantienen la misma posición que la manufactura. Los servicios resultan con los niveles educativos más elevados pues tienen la proporción mayor de microempresarios en los rubros de educación media-superior y superior, seguida de la manufactura y posteriormente el comercio. Que esto sea así puede deberse a las actividades características de cada sector, las respectivas habilidades, destrezas y conocimientos que requieren y las barreras a la entrada que los sectores pueden representar para los emprendedores. 


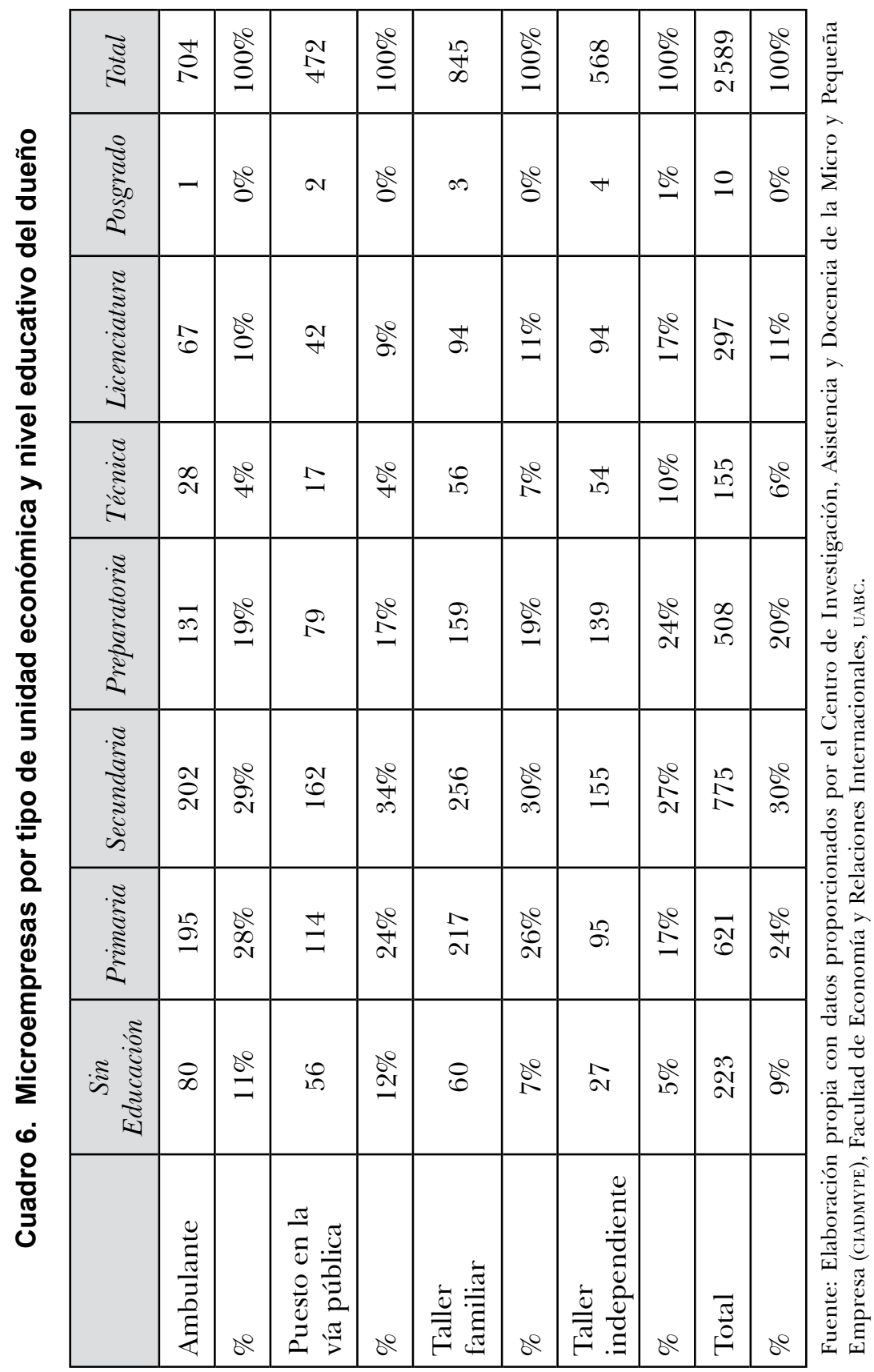


Estudios Fronterizos, nueva época, vol. 15, núm. 29, enero-junio de 2014

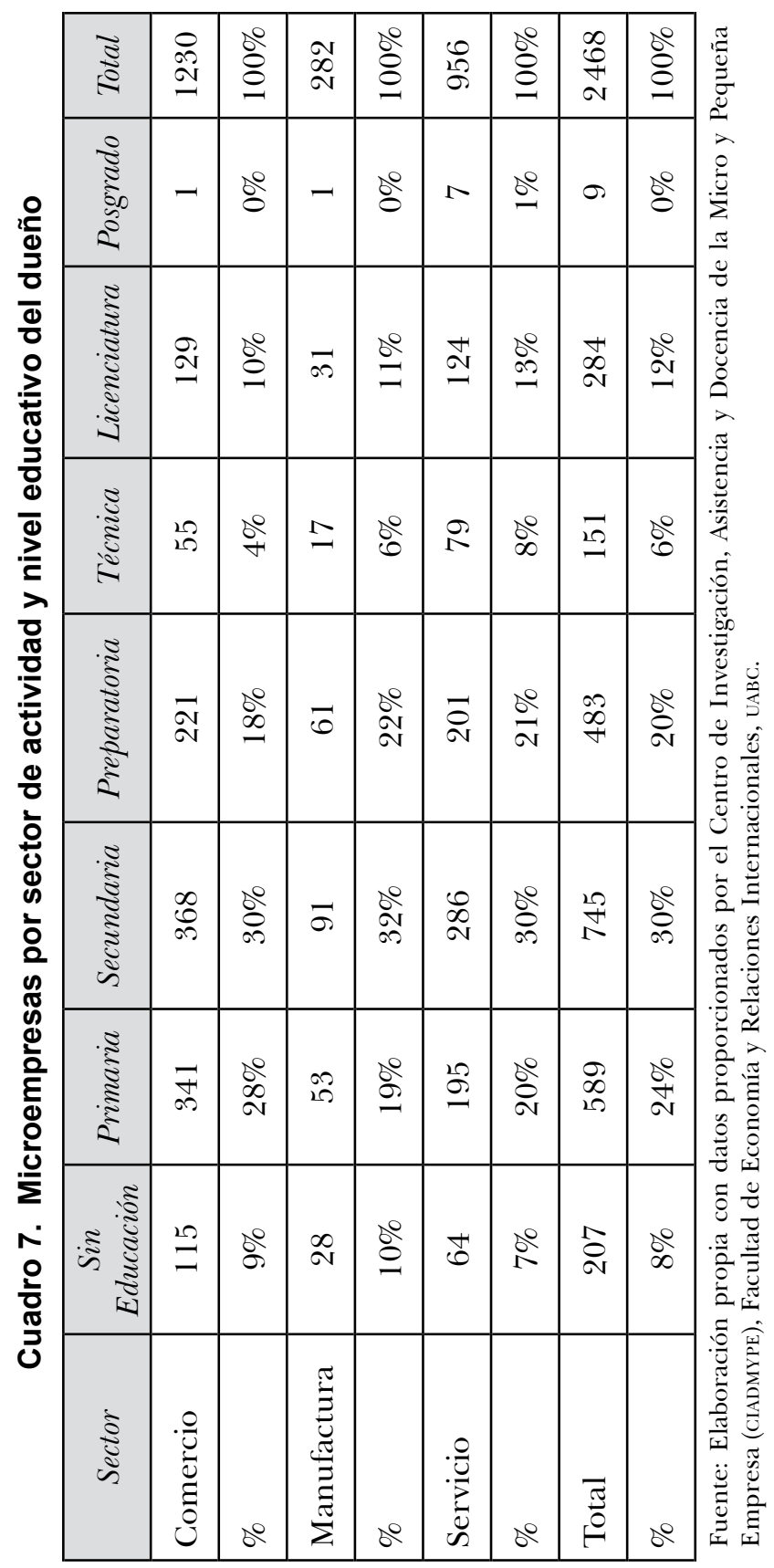




\section{Aprendizaje en las microempresas}

Experiencia en la operación

del negocio de los dueños por municipio

El tiempo en que la microempresa ha operado es una variable que aproxima la experiencia del dueño en el manejo del negocio. En el total de la muestra, se puede encontrar que $60 \%$ tiene una experiencia de un año o menos, cifra que es mayor para Ensenada y Mexicali, con $79 \%$ y $70 \%$ respectivamente; mientras que para Tecate y Tijuana la cifra llega a $62 \%$ y $55 \%$ (cuadro 8). Ello implica que dichas microempresas se encuentran en etapa de arranque o de desarrollo inicial. El caso de Playas de Rosarito contrasta pues cuenta con sólo $36 \%$ en este rango de experiencia. Se encuentra también $14 \%$ de las microempresas en el rango de más de un año y menos de tres, cifra que no presenta variaciones importantes entre

Cuadro 8. Microempresas por municipio y tiempo de operación en años

\begin{tabular}{|c|c|c|c|c|c|c|}
\hline & $0-1$ & $>1-3$ & $>3-5$ & $>5-10$ & $>10$ & Total \\
\hline Ensenada & 401 & 31 & 16 & 41 & 20 & 509 \\
\hline & $79 \%$ & $6 \%$ & $3 \%$ & $8 \%$ & $4 \%$ & $100 \%$ \\
\hline Mexicali & 607 & 97 & 48 & 66 & 50 & 868 \\
\hline & $70 \%$ & $11 \%$ & $6 \%$ & $8 \%$ & $6 \%$ & $100 \%$ \\
\hline P. Rosarito & 22 & 5 & 9 & 15 & 10 & 61 \\
\hline & $36 \%$ & $8 \%$ & $15 \%$ & $25 \%$ & $16 \%$ & $100 \%$ \\
\hline Tecate & 230 & 42 & 28 & 41 & 29 & 370 \\
\hline & $62 \%$ & $11 \%$ & $8 \%$ & $11 \%$ & $8 \%$ & $100 \%$ \\
\hline Tijuana & 1895 & 553 & 272 & 406 & 324 & 3450 \\
\hline & $55 \%$ & $16 \%$ & $8 \%$ & $12 \%$ & $9 \%$ & $100 \%$ \\
\hline Total & 3155 & 728 & 373 & 569 & 433 & 5258 \\
\hline & $60 \%$ & $14 \%$ & $7 \%$ & $11 \%$ & $8 \%$ & $100 \%$ \\
\hline
\end{tabular}

Fuente: Elaboración propia con datos proporcionados por el Centro de Investigación, Asistencia y Docencia de la Micro y Pequeña Empresa (CiADMYPE), Facultad de Economía y Relaciones Internacionales, UABC. 
municipios. A partir de los tres años de operación del negocio se piensa que las empresas superan la etapa crítica de desarrollo inicial, lo que mejora de forma importante las perspectivas de consolidación y permanencia en el mercado. En la muestra se puede detectar $26 \%$ de las empresas que han superado esta etapa, presentando algunas variaciones, particularmente para Playas de Rosarito y Tijuana, que cuentan con 56\% y 29\%, respectivamente, en esta etapa.

Fuentes de aprendizaje de las habilidades del negocio por municipio

Otra variable relevante en el capital humano son las fuentes de aprendizaje de las habilidades del negocio, lo cual se exhibe a detalle en el cuadro 9. Se observa que el autoaprendizaje es la fuente de conocimiento de las

\section{Cuadro 9. Microempresarios por fuente aprendizaje del negocio por municipio}

\begin{tabular}{|c|c|c|c|c|c|}
\hline & Autoaprendizaje & Escuela & $\begin{array}{c}\text { Parientes/ } \\
\text { amigos }\end{array}$ & $\begin{array}{c}\text { Trabajo } \\
\text { anterior }\end{array}$ & Total \\
\hline Ensenada & 223 & 58 & 101 & 77 & 459 \\
\hline$\%$ & $49 \%$ & $13 \%$ & $22 \%$ & $17 \%$ & $100 \%$ \\
\hline Mexicali & 415 & 108 & 209 & 74 & 806 \\
\hline$\%$ & $51 \%$ & $13 \%$ & $26 \%$ & $9 \%$ & $100 \%$ \\
\hline P. Rosarito & 9 & 1 & 9 & 7 & 26 \\
\hline$\%$ & $35 \%$ & $4 \%$ & $35 \%$ & $27 \%$ & $100 \%$ \\
\hline Tecate & 119 & 28 & 64 & 36 & 247 \\
\hline$\%$ & $48 \%$ & $11 \%$ & $26 \%$ & $15 \%$ & $100 \%$ \\
\hline Tijuana & 1098 & 150 & 593 & 212 & 2053 \\
\hline$\%$ & $53 \%$ & $7 \%$ & $29 \%$ & $10 \%$ & $100 \%$ \\
\hline Total & 1864 & 345 & 976 & 406 & 3591 \\
\hline$\%$ & $52 \%$ & $10 \%$ & $27 \%$ & $11 \%$ & $100 \%$ \\
\hline
\end{tabular}

Fuente: Elaboración propia con datos proporcionados por el Centro de Investigación, Asistencia y Docencia de la Micro y Pequeña Empresa (CiADMYPE), Facultad de Economía y Relaciones Internacionales, UABC. 
habilidades del negocio, lo cual se asocia ampliamente a la experiencia, por un lado, pero, por otro, también a la capacidad de aprender de los demás, que es inherente al ser humano, y que podría estar ocurriendo de forma espontánea en hogares y empresas. Ésta es la fuente de aprendizaje que poco más de $50 \%$ de los empresarios expresó haber tenido para su negocio. Esta fuente, junto con el aprendizaje obtenido de parientes y amigos que en total obtuvo $28 \%$, explica cerca del $80 \%$ de las respuestas de los microempresarios. No se observan diferencias importantes entre municipios, con excepción del municipio de Playas de Rosarito que tiene resultados más balanceados entre las fuentes, pues si bien el autoaprendizaje es importante (con 35\% de las respuestas), de forma equiparable lo es el aprendizaje de parientes y amigos (35\%) y el obtenido de trabajos anteriores $(27 \%)$. Es importante destacar la baja relevancia relativa de la escuela en todos los casos, particularmente Rosarito, y la aportación al aprendizaje de los trabajos anteriores, igualmente con excepción de este municipio. El primer caso puede explicarse por el tipo de formación recibida a nivel básico, donde se encuentra la mayoría de los microempresarios, que tiende a ser genérica y no tanto asociada a oficios, por lo que está desasociada del ramo de actividad del negocio. Las habilidades que la educación básica aporta en el individuo pueden ser aplicables a cualquier trabajo, incluyendo áreas de la microempresa. El segundo caso puede explicarse por el hecho de que muchas de las microempresas abren negocios que pudieran no tener que ver con las actividades de sus empleos anteriores, sino que más bien aprovecharon sus redes sociales para aprender y recibir asesoría sobre algún oficio o negocio, por lo que el trabajo anterior no figura como una fuente importante de aprendizaje.

\section{Fuentes de aprendizaje de las habilidades del negocio por sexo}

En lo que respecta a las fuentes de aprendizaje de las habilidades del negocio por sexo, el cuadro 10 ilustra de nueva cuenta la importancia del autoaprendizaje y de los parientes y amigos, aunque existen diferencias que vale la pena mencionar. En el caso de los hombres, el peso del autoaprendizaje es inferior (45\%) al de las mujeres (56\%), pero la aportación de trabajos previos es superior ya que el primer grupo posee $16 \%$ en esa 
Cuadro 10. Microempresarios por fuente aprendizaje del negocio y sexo

\begin{tabular}{|c|c|c|c|c|c|}
\hline & Autoaprendizaje & Escuela & Parientes/amigos & Trabajo anterior & Total \\
\hline Hombre & 395 & 69 & 282 & 141 & 887 \\
\hline & $45 \%$ & $8 \%$ & $32 \%$ & $16 \%$ & $100 \%$ \\
\hline Mujer & 759 & 139 & 344 & 119 & 1361 \\
\hline & $56 \%$ & $10 \%$ & $25 \%$ & $9 \%$ & $100 \%$ \\
\hline Total & 1154 & 208 & 626 & 260 & 2248 \\
\hline & $51 \%$ & $9 \%$ & $28 \%$ & $12 \%$ & $100 \%$ \\
\hline
\end{tabular}

Fuente: Elaboración propia con datos proporcionados por el Centro de Investigación, Asistencia y Docencia de la Micro y Pequeña Empresa (CiAdmyPe), Facultad de Economía y Relaciones Internacionales, UABC.

respuesta contra 9\% en el caso de las mujeres. Ello pudiera explicarse por las condiciones entre ambos sexos, pues las mujeres tienden a pasar más tiempo en casa y tomar mayor responsabilidad en el cuidado de los hijos, por lo que su exposición a empleos es menor y sus necesidades de autoaprendizaje mayores. El resto de las fuentes no presenta variaciones importantes entre los grupos.

Experiencia previa en los negocios

de los microempresarios por municipio

En algunos casos las experiencias previas a los negocios de los microempresarios son importantes porque determinan procesos de incubación que permiten adquirir conocimiento técnico favorable al desarrollo de la microempresa. En relación con la experiencia anterior de los microempresarios (cuadro 11), se observa que la mayoría de ellos eran empleados antes de ser emprendedores, al registrar más de $50 \%$ en todos los municipios. Ello puede explicarse porque el establecimiento de una microempresa es una forma de lograr independencia laboral o una fuente de ingreso alternativa en caso de desempleo. Esta tendencia se enfatiza en Rosarito al ser el municipio que muestra el mayor porcentaje (73\%). Las actividades 
Cuadro 11. Actividad anterior del microempresario por municipio

\begin{tabular}{|c|c|c|c|c|c|c|}
\hline & Hogar & Empleado & Estudiante & Microempresario & Otro & Total \\
\hline Ensenada & 109 & 268 & 45 & 39 & 9 & 470 \\
\hline & $23 \%$ & $57 \%$ & $10 \%$ & $8 \%$ & $2 \%$ & $100 \%$ \\
\hline Mexicali & 228 & 411 & 109 & 43 & 17 & 808 \\
\hline & $28 \%$ & $51 \%$ & $13 \%$ & $5 \%$ & $2 \%$ & $100 \%$ \\
\hline Rosarito & 4 & 19 & 2 & 1 & 0 & 26 \\
\hline & $15 \%$ & $73 \%$ & $8 \%$ & $4 \%$ & $0 \%$ & $100 \%$ \\
\hline Tecate & 73 & 133 & 15 & 21 & 5 & 247 \\
\hline & $30 \%$ & $54 \%$ & $6 \%$ & $9 \%$ & $2 \%$ & $100 \%$ \\
\hline Tijuana & 508 & 1217 & 159 & 173 & 36 & 2093 \\
\hline & $24 \%$ & $58 \%$ & $8 \%$ & $8 \%$ & $2 \%$ & $100 \%$ \\
\hline Total & 922 & 2048 & 330 & 277 & 67 & 3644 \\
\hline & $25 \%$ & $56 \%$ & $9 \%$ & $8 \%$ & $2 \%$ & $100 \%$ \\
\hline
\end{tabular}

Fuente: Elaboración propia con datos proporcionados por el Centro de Investigación, Asistencia y Docencia de la Micro y Pequeña Empresa (CIADMYPE), Facultad de Economía y Relaciones Internacionales, UABC.

del hogar son la segunda experiencia previa más representativa en las respuestas, siendo el municipio de Tecate el que muestra mayor porcentaje $(30 \%)$. Destaca el hecho de que los microempresarios han tenido en menor proporción experiencias previas como estudiantes y como microempresarios, por lo que los procesos de aprendizaje de las microempresas están relacionados con experiencias en empleos anteriores o actividades del hogar.

Experiencia previa en los negocios de los microempresarios por sexo

El análisis de la fuente de aprendizaje por sexo (cuadro 12) permite destacar que el ser empleado, como actividad anterior al establecimiento de 
Cuadro 12. Actividad anterior del microempresario por sexo

\begin{tabular}{|l|c|c|c|c|c|c|}
\hline & Hogar & Empleado & Estudiante & Microempresario & Otro & Total \\
\hline Hombre & 32 & 653 & 109 & 91 & 15 & 900 \\
\hline & $4 \%$ & $73 \%$ & $12 \%$ & $10 \%$ & $2 \%$ & $100 \%$ \\
\hline Mujer & 529 & 640 & 108 & 81 & 23 & 1381 \\
\hline & $38 \%$ & $46 \%$ & $8 \%$ & $6 \%$ & $2 \%$ & $100 \%$ \\
\hline Total & 561 & 1293 & 217 & 172 & 38 & 2281 \\
\hline & $25 \%$ & $57 \%$ & $10 \%$ & $8 \%$ & $2 \%$ & $100 \%$ \\
\hline
\end{tabular}

Fuente: Elaboración propia con datos proporcionados por el Centro de Investigación, Asistencia y Docencia de la Micro y Pequeña Empresa (CiADMyPe), Facultad de Economía y Relaciones Internacionales, UABC.

la microempresa, es mucho más frecuente en los hombres (73\%) que en las mujeres (46\%), presentándose el caso contrario en las actividades del hogar: hombres (4\%), mujeres (38\%). Ello puede deberse, por un lado, a que los hombres buscan actividades remuneradas pues, en algunos casos, son los que sostienen económicamente a la familia; y por otra parte, a que las mujeres están involucradas con actividades de la esfera laboral y del hogar en proporciones más equilibradas.

La condición de género no es determinante en actividades anteriores del microempresario, como estudiar o tener algún tipo de experiencia como empresario, ya que los porcentajes observados son similares tanto en hombres como en mujeres. Cabe destacar que tener alguna experiencia previa como empresario reporta el menor porcentaje. Por tanto, es posible que algunas estrategias de apoyo se relacionen con la provisión de servicios gratuitos para el progreso de negocios y fortalecimiento del capital humano no escolarizado.

\section{Capital humano y algunos indicadores de desempeño microempresarial}

Existen diversas variables que permiten analizar el desempeño de la microempresa; en este trabajo se consideran las ventas mensuales, el capital 
con el que la microempresa empezó a operar, el capital con el que la microempresa cuenta, el número de empleados, la relación ventas-empleados, la relación ventas-capital actual y la relación capital-empleados.

Como se puede observar en el cuadro 13, las ventas promedio de las microempresas fueron de 4400 pesos. Por nivel educativo se presenta la situación de que a mayor nivel de instrucción formal mayor es el monto de las ventas. De forma particular se observa que los microempresarios cuya educación es de preparatoria o superior, presentan en promedio mayores niveles de ventas que el promedio de la muestra y que los niveles educativos inferiores. Este mismo resultado se sostiene para el indicador del número de empleados, que sugiere que mayor educación brinda posibilidades de administrar microempresas más grandes. De la misa forma, existen impactos importantes de este tipo de capital humano sobre los niveles de eficiencia de las microempresas, particularmente si se mide como la relación ventas-empleados, que crece con el nivel de escolaridad del dueño del negocio. Las ventas-capital actual y capital-empleados parece no tener una relación con el nivel educativo, al menos con la base de datos utilizada.

Otra variable de desempeño del microempresario tiene que ver con los cambios en la organización, maquinaria y equipo y mejoras en el producto que se han realizado en la microempresa. En este caso, se observa una relación directa con el nivel de escolaridad del dueño. Es decir, la frecuencia con la que se realiza un cambio en la microempresa es mayor cuando el dueño cuenta con educación superior, dejando ver una tendencia clara de menor presencia de cambios en la microempresa a medida que el nivel de escolaridad es menor. En el cuadro 14 se muestra un resumen de los porcentajes de cambio o mejora de las microempresas. Se observa que los dueños de las microempresas sin educación, con educación básica y media superior, realizan cambios en cada caso en $21 \%$ de las microempresas, por lo que en $79 \%$ no hay evidencia de algún cambio. El porcentaje sube cuando se trata de educación superior, ya que en 33\% de los casos se realizaron cambios. En relación con los cambios en maquinaria y/o equipo en microempresas con dueños sin educación, solo $19 \%$ de éstas realizó cambios, siendo el porcentaje más alto para las microempresas con dueños que poseen educación superior (34\%).

En cuanto al rubro mejoras en el producto, es en el que con mayor frecuencia se realizan cambios. Al respecto, cabe destacar que en el rubro de 


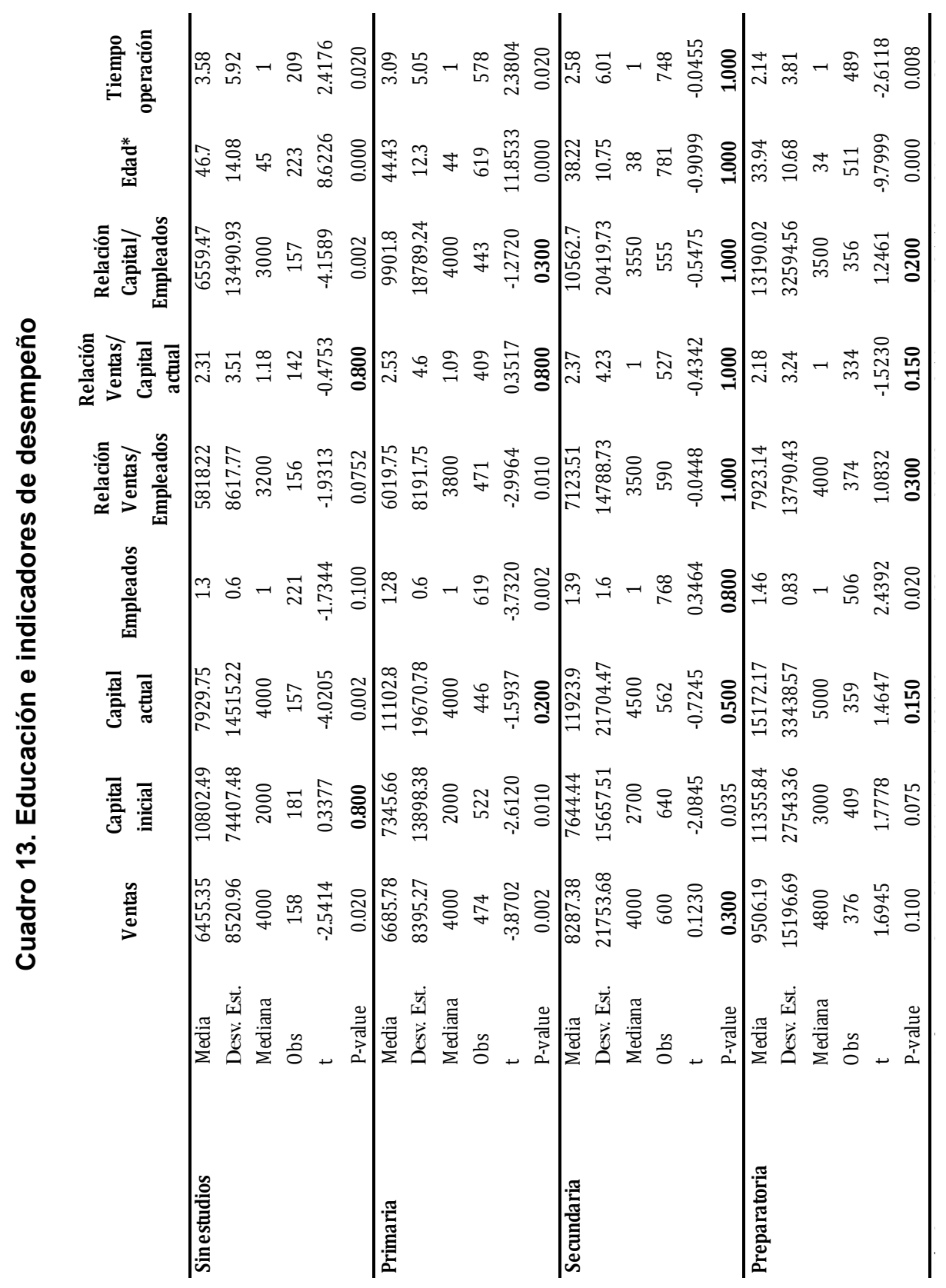




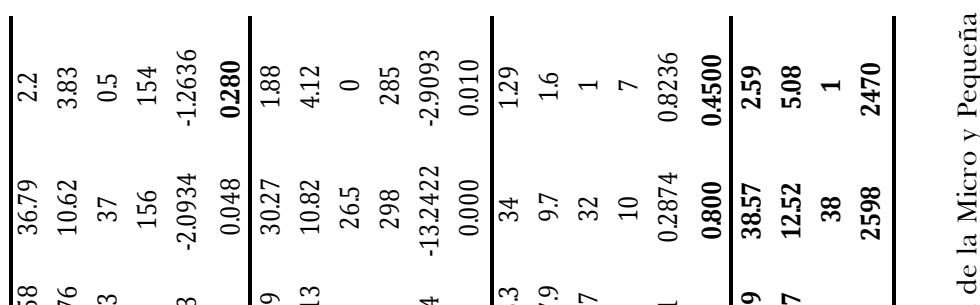

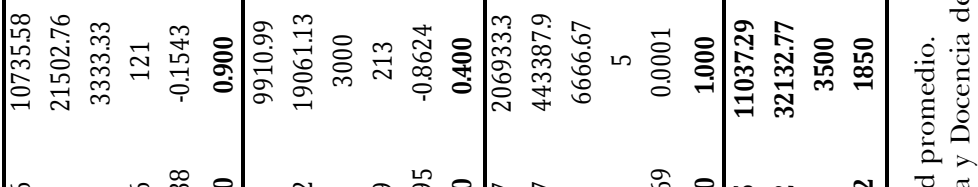
员

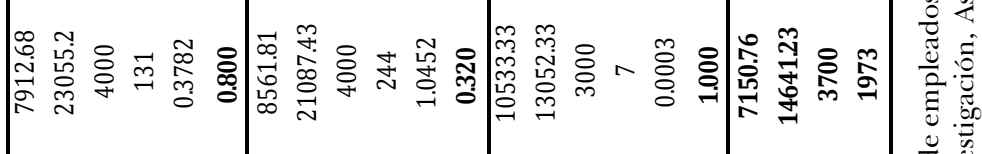
N

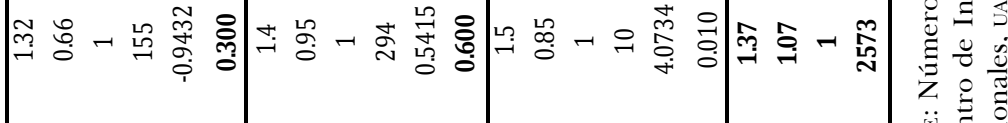
等

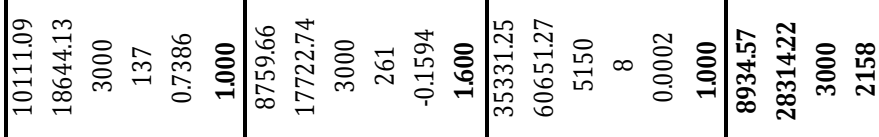

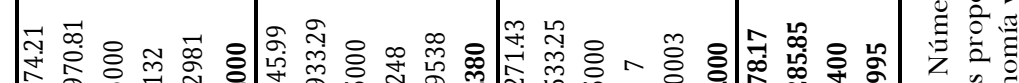

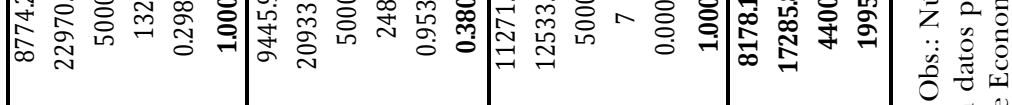

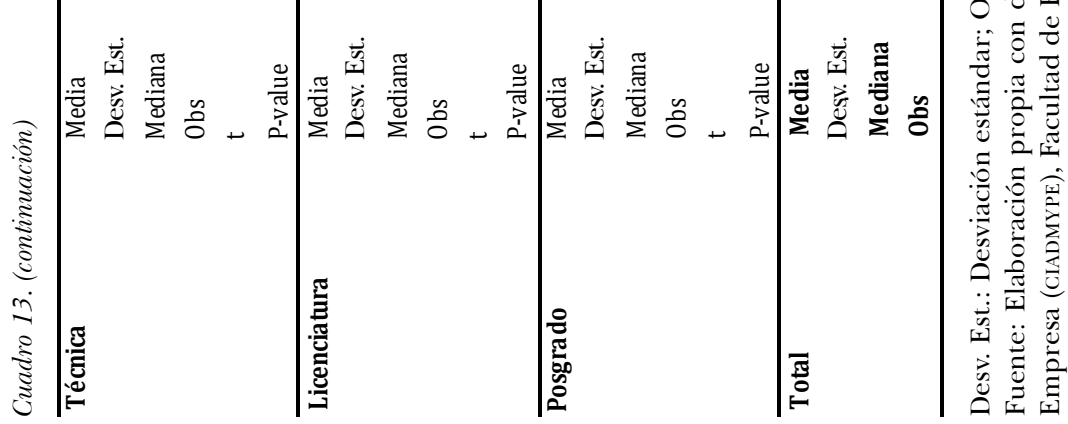


Cuadro 14. Cambios en la microempresa por nivel educativo del dueño

\begin{tabular}{|c|c|c|c|c|}
\hline & $\begin{array}{l}\text { Cambio en la } \\
\text { organización }{ }^{a}\end{array}$ & $\begin{array}{c}\text { Cambio en la } \\
\text { maquinaria y/o equipo }{ }^{a}\end{array}$ & $\begin{array}{c}\text { Mejoras en el } \\
\text { producto }^{a}\end{array}$ & Cambios $^{b}$ \\
\hline $\begin{array}{l}\text { Sin } \\
\text { educación }\end{array}$ & $21 \%$ & $19 \%$ & $34 \%$ & $25 \%$ \\
\hline E. básica & $21 \%$ & $24 \%$ & $39 \%$ & $28 \%$ \\
\hline $\begin{array}{l}\text { E. media } \\
\text { superior }\end{array}$ & $21 \%$ & $26 \%$ & $40 \%$ & $29 \%$ \\
\hline E. superior & $33 \%$ & $34 \%$ & $48 \%$ & $39 \%$ \\
\hline
\end{tabular}

${ }^{a}$ Los porcentajes indicados en cada columna corresponden a las microempresas que sí realizaron cambios.

${ }^{\text {b }}$ Promedio de los cambios en la organización.

Fuente: Ciadmype, con base en instrumento de diagnóstico.

Fuente: Elaboración propia con datos proporcionados por el Centro de Investigación, Asistencia y Docencia de la Micro y Pequeña Empresa (CIADMYPe), Facultad de Economía y Relaciones Internacionales, UABC.

dueños sin educación los cambios se hacen presentes en $34 \%$, el extremo es para el nivel de educación superior con $48 \%$. Ello puede deberse a que el entrenamiento formal contribuye al desarrollo de destrezas que conducen a un mejor desempeño. En particular, una mayor educación puede contribuir a que los microempresarios estén más enterados de los efectos positivos de los cambios organizacionales o tecnológicos, y a las mejoras del producto, pero también a que contribuye con una mayor capacidad de los emprendedores a leer y responder ante las circunstancias en los mercados.

Los indicadores de desempeño de las microempresas de la muestra relacionados con los años de experiencia del microempresario se muestran en el cuadro 15. El cuadro reporta en general la existencia de impactos favorables de los niveles de experiencia sobre los indicadores de desempeño. En cuanto a las ventas, puede observarse que los grupos de experiencia de 4 y 5 años, y más de 6 , presentan ventas por encima del promedio y de los grupos de entre 0 y 3 años de experiencia. El efecto de este tipo de capital humano es particularmente más importante en cuanto al número de empleados, lo que sugiere que a medida que el microempresario 


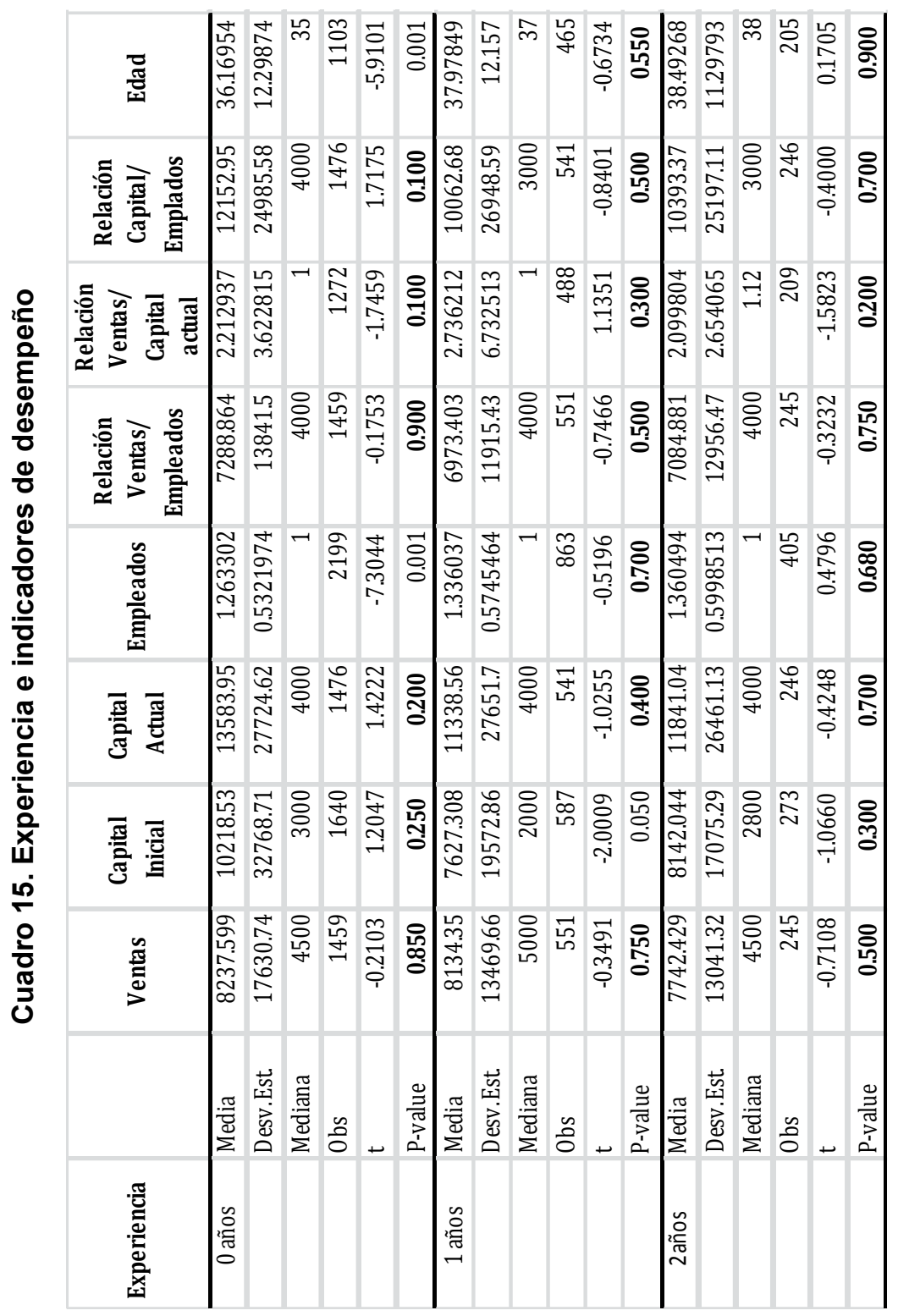


Estudios Fronterizos, nueva época, vol. 15, núm. 29, enero-junio de 2014

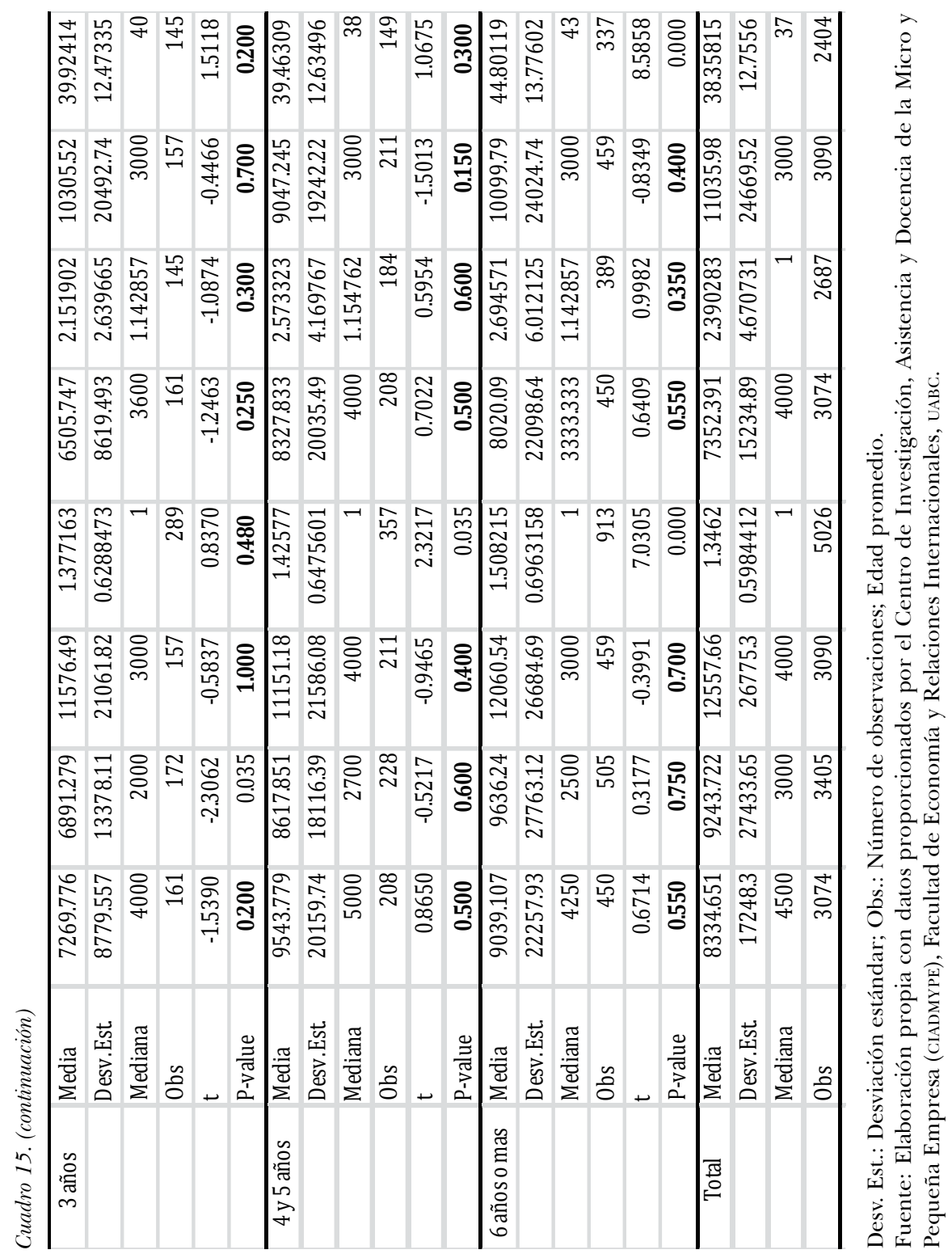


adquiere experiencia en el manejo del negocio, puede ir administrando mayor número de empleados. Ello sin duda también sugiere que la experiencia es un factor para la expansión de la empresa en términos de su tamaño, ya que en el rubro de 0 años, se emplean 1.26 empleados en promedio, cantidad que va incrementándose con la experiencia hasta llegar a 1.51, muy por encima del promedio de 1.35 trabajadores. Un resultado similar se encuentra para el indicador ventas-capital actual. Las relaciones ventas-empleados y capital-empleados, no presentan una relación clara con respecto de la experiencia.

\section{Pruebas de hipótesis}

En esta sección se realizan algunas pruebas de hipótesis para determinar estadísticamente el impacto del capital humano sobre variables de desempeño microempresarial. Esto se hace a partir de clasificar las microempresas por tipo y nivel de capital humano y después verificando las diferencias estadísticas que pudieran presentarse entre los grupos dependiendo de su perfil de capital humano. El ejercicio toma en consideración los grupos estratificados de acuerdo con los cuadros 13 y 15, que asocian el nivel educativo del dueño del negocio, por un lado, y la experiencia en la operación del negocio, por el otro. De esta forma se mide si los indicadores de desempeño microempresarial, en promedio, son estadísticamente iguales o diferentes respecto de los promedios por grupos de acuerdo con el nivel educativo y el nivel de experiencia. Los indicadores de desempeño considerados son las ventas mensuales, el capital inicial, el capital actual, el número de empleados, la relación ventas-empleados, la relación ventas-capital actual, y la relación capital-empleados, mismas que toman el valor de $X$ en este ejercicio. El ejercicio calculó el estadístico de prueba $t$ de Student y su respectivo $p$-value. La estimación del estadístico $t$ determina cuántas desviaciones estándar el grupo en cuestión está por arriba de la media. Por su parte, el p-value define la probabilidad de obtener un resultado al menos tan extremo como el que realmente corresponde. La hipótesis nula se representa de la siguiente manera:

$$
\text { Ho: } \bar{X}=\overline{\bar{X}}
$$


Donde, $\bar{X}$ es el valor promedio de la variable en análisis por cada una de las categorías, y $\bar{X}$ es valor promedio de las medias de cada una de las categorías. Como se indicó antes, estas categorías de capital humano son sin estudios, primaria, secundaria, preparatoria, técnica, licenciatura y posgrado; y las de aprendizaje por experiencia en la administración del negocio son $0,1,2,3$, entre 4 y 5 y más de 6 años de operación del negocio. Dado que el p-value nos permite evaluar la credibilidad de la hipótesis nula, cuanto más pequeño es su valor, menos probable es que Ho sea verdadera, por ello, si es menor a $0.05, \alpha=5 \%$, la hipótesis nula se rechaza. Los resultados de este ejercicio se encuentran en los cuadros 13 y 15 .

De acuerdo con los resultados de la cuadro 13, en las categorías de sin estudios y primaria, se observa que las medias de las variables analizadas son estadísticamente distintas al valor de la media del sector microempresarial en su conjunto. Por ejemplo, en el caso del grupo de microempresarios sin estudios y primaria, la prueba de hipótesis muestra que existe evidencia suficiente para concluir que las ventas son diferentes al promedio de las microempresas en observación, lo que de otra forma significa que el indicador es mayor para los grupos de secundaria o nivel educativo mayor. Este resultado aparece con una claridad similar para el capital inicial, donde se encuentra para los grupos de primaria, secundaria, técnica y preparatoria, que son significativamente más bajos que el promedio de toda la muestra. El indicador de capital actual también sugiere que al menos el grupo de sin estudios presenta un nivel inferior al de la muestra de microempresas en conjunto. Ello apunta a concluir una significancia estadística del impacto del nivel de educación sobre las ventas y el capital con el que la empresa opera. En cuanto al número de empleados, que refleja no sólo habilidades para la administración de negocios, sino también el tamaño de la empresa se encuentra con significancia estadística, que los grupos con dueños sin educación y educación primaria, cuentan con un número de empleados inferior al del promedio de la muestra; y que aquellos con nivel de posgrado presentan más empleados en promedio que el de la muestra en su conjunto. El grupo de microempresas con nivel de preparatoria también se encontró una presencia de un número de empleados superior al del promedio. Por último, se tienen los resultados para los indicadores de eficiencia que indican ciertos impactos interesantes. Por un lado, se encuentra que la relación ventas-empleados es significativa- 
mente inferior para los grupos de microempresas con dueños sin estudios (significancia estadística de 0.075 ) y con educación primaria que para la muestra de microempresas en su conjunto. De la misma forma, se encuentra que la relación capital-empleados es significativamente inferior para el grupo con dueños sin estudios que para la muestra en su conjunto. Estos datos sugieren que la educación es importante para la asignación de los recursos en las microempresas con el resultado de mayores niveles de eficiencia y también de mejores condiciones de capitalización por empleado.

En el caso del cuadro 15 que contiene los resultados para el análisis del aprendizaje por experiencia en la operación del negocio. La mayoría de las pruebas muestran evidencia suficiente de que los valores medios son iguales a los valores de la muestra total. Lo anterior indica que cuando se estratifican las microempresas de acuerdo al nivel de experiencia del dueño en la operación del negocio como indicador de capital humano, no se encuentran diferencias estadísticas entre los grupos con respecto a las variables de desempeño en general. Sin embargo, solo en cuanto al capital inicial promedio y el número de empleados, se encuentra evidencia de diferencias entre grupos. En el primer caso, las empresas con solo 1 año de operación tuvieron un capital inicial inferior al del conjunto de la muestra. En el segundo caso, en cuanto el número de empleados, el estudio sugiere que las empresas en promedio son más pequeñas en el grupo de 0 años de operación, y más grandes en los grupos de 4 y 5 y más de 6 años de operación, que el promedio de la muestra de microempresas en su conjunto.

\section{Conclusiones}

Este trabajo se orientó a conocer la estructura del capital humano y el aprendizaje en las microempresas de base social del estado de Baja California, y a determinar el papel de éste sobre algunos indicadores de desempeño microempresarial. Con ello, el documento pretende establecer un diagnóstico sobre la contribución de este recurso y obtener algunas implicaciones de política pública para el sector. Los resultados descriptivos del trabajo apuntan hacia la caracterización del sector de microempresas de base social para el estado: los dueños de una microempresa típica cuenta con estudios de secundaria o inferior (63\%), operan microempresas que 
en promedio llevan 3.18 años subsistiendo, y la mayoría ha operado por un año o menos. Esta caracterización es similar entre hombres y mujeres, aunque las condiciones de los hombres en cuanto a educación son ligeramente más favorables. Según los resultados, el 79\% del sector de microempresarios obtuvo sus aprendizajes del negocio a través del autoaprendizaje o de parientes o amigos, sólo $10 \%$ los obtuvo en la escuela y $11 \%$ en trabajos anteriores. Ello a pesar de que $56 \%$ de los microempresarios dijeron haber sido empleados en su actividad anterior, $8 \%$ microempresarios y $9 \%$ estudiantes; en el grupo de las mujeres destaca que el $38 \%$ trabajaba en el hogar, antes de transitar al emprendimiento.

En cuanto al papel del capital humano sobre el desempeño de los negocios y las capacidades empresariales, se encontró que éstas desempeñan un papel importante en el tipo de microempresa, ya que del total de microempresas de tipo ambulante o puesto en la vía pública, $68 \%$ y $70 \%$ respectivamente son operados por individuos sin educación o con secundaria como máximo; para los talleres familiares e independientes estas cifras son de $63 \%$ y $49 \%$ respectivamente. De igual forma, se encontró que el nivel de escolaridad impacta sustancialmente la disposición a impulsar cambios en la microempresa, tanto organizacionales, de maquinaria y equipo, como de producto. Con significancia estadística también se encontró que el nivel de escolaridad impacta el nivel de ventas de los micronegocios, su tamaño medido por el número de empleados, y los niveles de eficiencia si se mide como la relación ventas-empleados. Respecto de la experiencia medida en años de operación del negocio, la mayoría de las pruebas muestra evidencia suficiente de que los valores medios son iguales a los valores de la muestra total; sólo en cuanto al capital inicial promedio y el número de empleados se encuentra evidencia de impacto.

Los resultados afirman la importancia del capital humano sobre el desempeño de este tipo de negocios, pues apoyan lo que se encuentra regularmente en la literatura para las grandes empresas o sectores en cuanto a la relación entre capital humano y el desempeño medido desde diversas perspectivas. Ello es particularmente cierto para la educación formal. Los resultados también dan cuenta no sólo de la baja escolaridad de quienes operan este tipo de negocios, sino también de su bajo aprovechamiento como fuente de aprendizaje para impulsar sus negocios. Los resultados ilustran, asimismo, la heterogeneidad en la distribución del capital huma- 
no microempresarial entre localidades, sectores, tipos de empresas y género. Ambos grupos de resultados deben servir para el diseño de políticas públicas y diferenciar los tipos de acciones.

Algunas implicaciones de política pública surgen del trabajo, algunos directamente relacionados con sus hallazgos, que justifican algunas de las recomendaciones y otras que no son nuevas, pero continúan siendo relevantes en virtud de los resultados del trabajo. Derivado de las prescripciones generales desde la literatura del desarrollo, las políticas orientadas a aumentar la cobertura educativa y aumentar el nivel de escolaridad promedio de las personas ayudaría a ampliar la dotación inicial de este importante recurso mejorando el desempeño microempresarial, y en general las capacidades humanas para el trabajo productivo en otras empresas. En particular, las acciones encaminadas a reducir el analfabetismo y la deserción a partir de fomentar el tránsito de los estudiantes de la educación primaria y secundaria hacia la media superior y superior, adquieren gran pertinencia. Esto es importante porque muchos estudiantes dejan de estudiar en el nivel de secundaria y transitan hacia el emprendimiento microempresarial. Un mayor nivel de escolaridad en este tipo de negocios daría un impulso inicial más fuerte de sus empresas con resultados favorables sobre su desempeño. Además del avance de la educación para aumentar la cobertura, los vínculos de los estudiantes con los aspectos empresariales serían también relevantes. Una manera de fortalecer el aprendizaje formal y mejorar el impacto de la educación sobre los aprendizajes de los microempresarios y su desempeño, sería la ejecución de programas destinados a la transferencia de capital humano, particularmente la prestación de servicios de desarrollo empresarial. En los países en desarrollo, la implementación de este tipo de educación es factible a través de programas de extensión utilizando metodologías adecuadas o la incorporación de algunas herramientas y conceptos básicos de negocios en los currículos de estudio en la secundaria. Este tipo de políticas pueden dar no sólo capacitación a los estudiantes antes de su tránsito a la actividad microempresarial, inevitable por sus condiciones socioeconómicas, sino también promover la cultura empresarial antes y en apoyo a niveles educativos superiores. La educación organizada de distintas formas puede contribuir a fortalecer las capacidades de aprendizaje de los microempresarios para que capitalicen efectivamente la ex- 
periencia acumulada en la propia microempresa, en otras experiencias microempresariales, y en empleos anteriores, fomentando de esa manera sus capacidades de emprendimiento.

Finalmente, se encuentra bien establecido en la literatura que los emprendimientos de base social son inherentes a los individuos y a las familias con bajo nivel de capital humano, imposibilitados a incursionar en los mercados laborales por su baja capacitación y habilidades. También está ampliamente reconocido que la incidencia de este tipo de emprendimientos se relaciona con las condiciones de desarrollo en las regiones y los países. Es por ello que políticas fuertemente orientadas al desarrollo, como lo son las políticas educativas, son de la mayor pertinencia. Políticas educativas que promuevan una mayor cobertura, impacto formativo y mayores capacidades productivas en los individuos, permitirán, por un lado, que éstos tengan mejores oportunidades de empleo y expectativas salariales, incentivando el cierre de sus negocios de subsistencia; por otro lado, permitirán también que los emprendimientos que se generen verdaderamente aprovechen oportunidades de mercado de forma innovadora y, consecuentemente, con condiciones de crecimiento más favorables. Es por ello que la educación debe constituirse siempre en el pilar de cualquier política pública para el desarrollo.

\section{Bibliografía}

Aguilar, J. G., Ramírez, N. y Barrón K. (2007). Conformación de la microempresa marginada en la frontera norte de México. Estudios Fronterizos, VIII(15), 51-71. Altonji, J. y Dunn, T. (1991). Relationships among the family incomes and labor outcomes of relatives. En: R. G. Ehrenberg Research. Labor Economics, (pp. 269-310). Greewich: JaI Press.

Arrow, K. (1962). The economic implication of learning by doing. Review of Economics Studies, 29, 155-173.

BID (Banco Interamericano de Desarrollo). (1997). Desarrollo de la microempresa: Estrategia sectorial.

BID (Banco Interamericano de Desarrollo). (2005). El Grupo вID y la microempresa (1990-2005): Resultados de 15 años de Trabajo.

Barron, J., Black, D. y Loewenstein, M. (1987). Employer size: The implications for search, training, capital investment, starting wages, and wage growth. Journal of Labor Economics, 5(1), 76-89. 
Martín Ramírez Urquidy, Michelle Texis Flores y José Gabriel Aguilar Barceló/El papel del capital humano

Bartel, A. (1989). Formal employee training programs and their impact on labor productivity: Evidence from a human resource survey. (Documento de Trabajo núm. 3026). Cambridge, Massachusetts: National Bureau of Economics Research.

Bartel, A. (1992). Productivity gains from the implementation of employee training programs. (Documento de Trabajo núm. 3893). Cambridge, Massachusetts: National Bureau of Economics Research.

Bates, T. (1987). Self-employed minorities: Traits and trends. Social Sciences Quarterly, 68, 539-550.

Bates, T. (1990). Entrepreneur human, capital inputs and small business longevity. Review of Economics and Statistics, 72(4), 551-559.

Becker, G. S. (1975). Human capital: A theoretical and empirical analysis with special reference to education ( $2^{\mathrm{da}} \mathrm{ed}$.). Nueva York: Nber.

Becker, G. S., (1962). Investment in human capital: A theoretical analysis. The Journal of Political Economy, 70(5), 9-49.

Berger, M. S. (1997). Microenterprise development strategy. Washington: BID.

Bishop, J. (1994). The impact of previous training on productivity and wages. En L. Lynch (Ed.), Training and the private sector: International comparisons, (pp. 99161). Chicago: University of Chicago Press.

Black, S. y Lynch, L. (1996). Human capital investment and productivity. Documentos y procedimientos de Hundredth and Eighth Annual Meeting of the American Economic Association San Francisco. The American Economic Review, 86(2), 263- 267.

Carpintero, S. (1998). Los programas de apoyo a la microempresa en América Latina. Bilbao: Ediciones Deusto.

Dunn, T. y Holtz-Eakin, D. (2000). Financial capital, human capital, and the transition to self-employment: Evidence from intergenerational links. Journal of Labor Economics, 18(2), 282-305.

Fane, G. (1975), Education and the managerial efficiency of farmers. Review of Economics and Statistics, 57(4), 452-461.

Huffman, W. E. (1977). Allocative efficiency: The role of human capital. Quarterly Journal of Economics, 91(1), 59-79.

Jovanovic, B. y Nyarco, Y. (1995). A bayesian learning model fitted to a variety of empirical learning curves. Brooking Papers of Economic Activity, 1995, 247-305.

Laferrère A. y McEntee, P. (1996). Self-employment and intergenerational transfers: Liquidity constraint or family environment? (Documento de Trabajo núm. 9637), París: Centre de Recherche en Economie at Statistque.

Liedholm, C. (2002). Small firm dynamics: Evidence from Africa and Latin America. Small Business Economics, (18), 227-242.

Hernández-Trillo, F., Pagán J. A. y Paxton, J. (2005). Start-up capital, microenterprises and technical efficiency in Mexico. Review of Development Economics, 9(3), 434-447. 
Mungaray, A. y Ramirez-Urquidy, M. (2007). Capital Humano y Productividad en microempresas. Investigación Económica, LXVI(260), 81-115.

Mungaray, A., Ramírez-Urquidy, M., Texis, M., Ledezma D. y Ramírez, N. (2008). Learning economics by servicing: A mexican experience of service learning in microenterprises. International Review of Economics Education, 7(2), 19-38.

Mungaray, A., Ramírez, N. y Texis, M. (2005). Estructura de mercado y maximización de beneficios en las microempresas. Comercio Exterior, 55(4), 316-321.

Parker, S. y Robson, M. (2004). Explaining international variations in selfemployment: Evidence from a panel of OECD Countries. Southern Economic Journal, 71(2), 287-301.

Ruiz, C. (1995). Economía de la pequeña empresa. México: Ariel.

Shultz, T. (1960). Capital formation by education. Journal of Political Economy, 68, $571-583$.

Shultz, T. (1961). Investment in human capital. American Economic Review, 51(1), $1-17$.

Shultz, T. (1972). Investment in human capital. Education and the economics of human capital. Nueva York: Ronald Wykstra, The Free Press.

Stefanou, S. E. y Saxena, S. (1988). Education, experience and allocative efficiency: A dual approach. American Journal of Agricultural Economics, 7(2), 338-345.

Teplitz, C. (1991). The learning curve deskbook, a reference guide to theory, calculations, and applications. Nueva York: Quorum Books.

Texis, F. M., Mungaray, A., Ramírez-Urquidy, M. y Ramírez, N. (2011). Aprendizaje en microempresas de Baja California. Estudios Fronterizos. Nueva Época, 12(23), 95-116.

Young, R., Francis, D. y Young, C. (1993). Innovation, high-technology use and flexibility in small manufacturing firms. Growth and Change, 24(1), 67-86. 